\title{
An adaptive interpolation scheme for molecular potential energy surfaces
}

\author{
Markus Kowalewski, ${ }^{1}, 2$, a) Elisabeth Larsson, ${ }^{1}$ and Alfa Heryudono ${ }^{3}$ \\ 1) Department of Information Technology, Uppsala University, Box 337, SE-751 05 Uppsala, \\ Sweden \\ ${ }^{2)}$ Now at: Chemistry Department, University of California, Irvine, California 92697-2025, \\ United States \\ 3) Department of Mathematics, University of Massachusetts Dartmouth, Dartmouth, Massachusetts 02747, \\ United States
}

The calculation of potential energy surfaces for quantum dynamics can be a time consuming task - especially when a high level of theory for the electronic structure calculation is required. We propose an adaptive interpolation algorithm based on polyharmonic splines (PHS) combined with a partition of unity (PU) approach. The adaptive node refinement allows to greatly reduce the number of sample points by employing a local error estimate. The algorithm and its scaling behavior is evaluated for a model function in 2, 3 and 4 dimensions. The developed algorithm allows for a more rapid and reliable interpolation of a potential energy surface within a given accuracy compared to the non-adaptive version.

\section{INTRODUCTION}

Molecular potential energy surfaces (PES) are frequently used in theoretical chemistry to solve the Schrödinger equation for the nuclei. The task of calculating those PESs is routinely carried out by quantum chemistry (QC) programs for e.g., the calculation of infrared spectra. The PES is represented in the simplest possible way by harmonic oscillators through the second derivatives of the energy with respect to the nuclear coordinates. While this method is straightforward, for any method that goes beyond a harmonic approximation there are some different options. High resolution spectroscopy and chemical reaction dynamics rely on a global representation of the PES, often realized using analytical functions ${ }^{1}$. While analytical functions are an efficient way to describe molecular systems around an equilibrium geometry, reaction dynamics explores configurations far off the equilibrium structures.

The solution of the time dependent Schrödinger equation (TDSE) for reaction dynamics usually relies on the Born-Oppenheimer approximation ${ }^{2}$, meaning that the PES for solving the the TDSE in the space of the nuclear degrees of freedom is obtained from quantum chemistry methods. The shape of the potential energy landscape can become fairly complex, which can make the use of analytical functions quite difficult. In principle one could calculate the needed grid by directly calculating every point with an ab initio method. However, this is usually too expensive since a quantum dynamics simulation based on Fourier or finite difference methods requires a fairly dense grid of sample points?

The two main categories for the representation of PESs can be divided into fitting methods and interpolation methods. Fitting methods include simple polynomial representations, many-body polynomials 3 and a broad variety of neural networks 4 . Interpolation methods pro-

\footnotetext{
a) Electronic mail: mkowalew@uci.edu
}

vide the possibility to represent the PES without the necessity of prior knowledge of its shape, and reproduce the function exactly at the sample points. Interpolation methods based on Shepard's method $\sqrt{6}\left[\frac{8}{8}\right.$ perform well, when combined with energy gradients and second derivatives. Interpolating moving least squares ${ }^{9 \mid 10}$ target efficient and automated calculation of PESs. Other interpolation methods which have been applied for PESs include cubic splines ${ }^{11112}$, Hilbert space reproducing kernel ${ }^{13}$, which are a radial basis function (RBF) variant, or are specially designed for inter-surface crossings 14 . A very general approach for two-dimensional surfaces is the thin plate spline interpolation ${ }^{15}$ which has been successfully applied to the quantum dynamics of reactive systems 16117 . However, already in three dimensions the number of sample points can become large enough to make the solution of the underlying linear system of equations computationally overly expensive.

In this paper we present an interpolation approach which is driven by the practical need for an efficient interpolation scheme within a few dimensions in combination with high level QC methods (e.g. MPn, coupled cluster, configuration interaction $\left.{ }^{18}\right)$. The main objectives motivating the development are: The minimization of the number of sample points necessary to obtain an interpolant of given accuracy. This reduces the number of ab initio calculations, since those can take up a major amount of the calculation time. The scheme should should avoid the $\mathcal{O}\left(N^{3}\right)$ scaling behavior of linear solvers and thus be able to handle large numbers of sample points efficiently. The scheme should yield reliable PESs by providing error control without prior knowledge of the shape of the PES. Moreover, it should not rely on the use of energy gradients or second derivatives, which might not available or too expensive for some high-level QC methods. The approach we use is based on polyharmonic splines (PHSs) ${ }^{15}$ in combination with a PU approach ${ }^{19}$ to allow for a larger number of sample points. The PHSs can be set up such that they provide smooth surfaces by avoiding oscillatory behavior in between sample points. An iterative refinement scheme reduces the number of 
sample points and allows for setting an error tolerance.

The aim of this paper is to provide a scalable method for a rapid and reliable interpolation of low dimensional PESs solely based on single point energy calculation without the necessity of prior knowledge of its qualitative features.

\section{METHOD}

In the following the building blocks for the algorithm will be discussed. In sec. II A we briefly review the PHS interpolation and its combination with a PU scheme. In sec. IIB the algorithm for the local refinement scheme is introduced and then followed by an experimental analysis of the algorithm's performance in sec. III.

\section{A. The interpolation scheme}

The basic interpolation method has to be chosen such that it fits the properties of the function it should approximate. In the absence of avoided crossings and conical intersections PESs are smooth functions with continuous derivatives. This paper will focus on the case where the potential is represented by a smooth function. Cusps and other features involving discontinues in the higher derivatives need special attention which is beyond the scope of this paper. Thin plate splines 20121 have been used successfully in the past to reliably interpolate two-dimensional PESs from a coarse grid obtained by $\mathrm{QC}$ calculations to much finer grids as needed for TDSE calculations. thin-plate splines were originally designed to minimize the bending energy of a sheet of metal forced out of plane configuration at a certain number of points. Mathematically this is achieved by minimizing the norm over the second derivatives of the interpolant. This avoids oscillatory behavior in between the sample points, which is a basic requirement for a robust interpolation scheme suitable for PESs. The generalization of thin-plate splines to higher dimensions and to higher orders are the poly harmonic splines (PHS) $\sqrt{15}$. The properties, of minimizing the bending energies of second order and higher, is preserved thus avoiding oscillatory behavior also in higher dimensions than two. The general PHS interpolant $u(\mathbf{x})$ is given by:

$$
u(\mathbf{x})=\sum_{j=1}^{N} \lambda_{j} \phi_{j}^{m}(\mathbf{x})+\mathcal{P}^{m}(\mathbf{x}),
$$

where $u$ is defined by a set $X=\left\{\mathbf{x}_{c, 1}, \ldots, \mathbf{x}_{c, N}\right\}$ of $N$ sample points and $\mathbf{x}=\left(x_{1}, \ldots, x_{d}\right)$ is a vector in $\mathbb{R}^{d}$. The basis functions of a PHS are conditionally positive definite RBFs $\phi_{j}^{m}(\mathbf{x})$ of order $m$ of the form:

$$
\phi_{j}^{m}(\mathbf{x}) \equiv \phi^{m}\left(\left\|\mathbf{x}-\mathbf{x}_{j}\right\|_{2}\right)= \begin{cases}r^{2 m-2} \ln (r) & d \text { even } \\ r^{2 m-1} & d \text { odd }\end{cases}
$$

Here $r$ is defined by the euclidean distance $\|\cdot\|_{2}$. The PHS also includes a multivariate polynomial term

$$
\mathcal{P}^{m}(\mathbf{x})=\sum_{|p|=0}^{m-1} \alpha_{p} \mathbf{x}^{p}
$$

where $p=\left(p_{1}, \ldots, p_{d}\right)$ is a multi-index of positive integers $\mathbb{N}_{0}^{+}$such that $|p|=p_{1}+\cdots+p_{d}$ and $\alpha_{p}$ are the polynomial coefficients. As an example, for a polynomial with order $m=3$ and dimension $d=2$ this corresponds to the multivariate second order polynomial

$$
\mathcal{P}^{3}(\mathbf{x})=\alpha_{1}+\alpha_{2} x_{1}+\alpha_{3} x_{2}+\alpha_{4} x_{1}^{2}+\alpha_{5} x_{1} x_{2}+\alpha_{6} x_{2}^{2} .
$$

The coefficients $\lambda$ and $\alpha$ defining the interpolant from eq. (1) and (3) are obtained by solving the linear system

$$
\left(\begin{array}{cc}
A & P \\
P^{T} & 0
\end{array}\right)\left(\begin{array}{l}
\lambda \\
\alpha
\end{array}\right)=\left(\begin{array}{l}
f \\
0
\end{array}\right) \text {. }
$$

The matrix elements of $A^{N \times N}$ represent the RBFs and are defined as $A_{i j}=\phi^{m}\left(\left\|\mathbf{x}_{i}-\mathbf{x}_{j}\right\|_{2}\right)$. The vector $f=\left(f_{1}, \ldots, f_{N}\right)$ contains the function values $f\left(\mathbf{x}_{j}\right)$ at the sample points $\mathbf{x}_{j}$. The polynomimal part of (1) is represented by the block $P^{N \times s}$ :

$$
P=\left(\begin{array}{cccc}
1 & \mathbf{x}_{1}^{p_{1}} & \ldots & \mathbf{x}_{1}^{p_{s-1}} \\
\vdots & \vdots & & \vdots \\
1 & \mathbf{x}_{N}^{p_{1}} & \ldots & \mathbf{x}_{N}^{p_{s-1}}
\end{array}\right)
$$

The number of columns in $P$ are given by the binomial coefficient $s=\left(\begin{array}{c}m-1+d \\ d\end{array}\right)$. Thus a linear problem of size $N+s$ has to be solved. Due to the polynomial part of eq. (1) a polynomial of degree $m-1$ can be represented exact by the interpolant.

The PHS interpolant from eq. (1) can already be used for the approximation of a PES with a moderate number of sample points. However, the number of sample points that can be used in a practical scenario is limited by its scaling behavior. For dense coefficient matrices, linear solvers usually scale with $\mathcal{O}\left(N^{3}\right)$ meaning that with a large number of sample points, the computational time can quickly become too large. Also, for a large number $N$ the condition number of $A$ increases and the linear system becomes ill conditioned, thus posing numerical problems. A solution to this problem is to break up the global nature of eq. (1) by splitting up the interpolation domain in sub-domains of smaller sizes which can then be handled independently in the PHS interpolation.

There are several different methods available allowing for localization. Compactly supported RBFs can be used to introduce a sparsity pattern in $A$, but there is a tradeoff between sparsity and accuracy ${ }^{15}$ (Chapter 12), and the support radius parameter needs to be carefully adjusted. Moreover, a different choice of RBFs might interfere with the basic requirements to avoid oscillatory behavior. A fast multipole expansion is another powerful method which can be used for a fast evaluation of RBF 


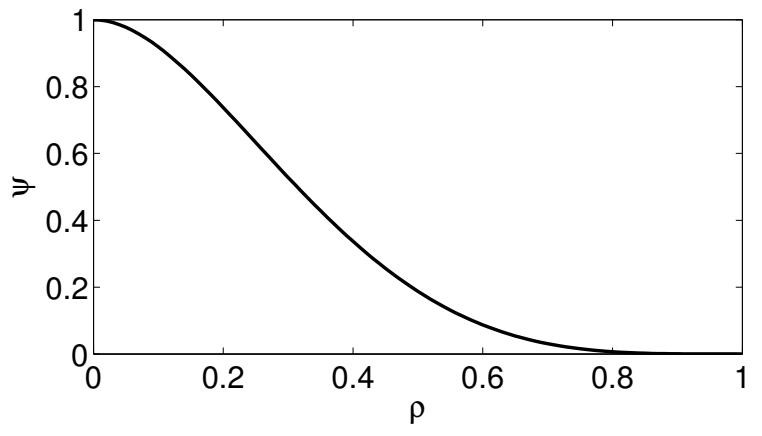

FIG. 1. Illustration of Wendland's $C^{2}$ function from eq. (9) for two and three dimensions.

interpolants but requires a large number of points to pay ofl 22. A stable and simple to implement method is the so called PU method 19 , which can be understood as generalization of Shepard's interpolation method 6 . The interpolation domain is covered by $N_{P}$ overlapping $(d-$ dimensional) patches. The global interpolant $s(\mathbf{x})$ is then given by the weighted sum over all $N_{P}$ local patches.

$$
s(\mathbf{x})=\sum_{i=1}^{N_{P}} w_{i}(\mathbf{x}) u_{i}(\mathbf{x}),
$$

The local interpolant $u_{i}(\mathbf{x})$ for every patch is a PHS interpolant (see eq. (1)). The PU weights $w_{i}(\mathbf{x})$ are then found by using Shepard's method.

$$
w_{i}(\mathbf{x})=\frac{\psi_{i}(\mathbf{x})}{\sum_{l=0}^{N_{P}} \psi_{l}(\mathbf{x})}
$$

The generating functions $\psi(\mathbf{x})$ used here are Wendland's $C^{2}$ functions ${ }^{25}$ with compact support:

$$
\psi_{i, d}(\mathbf{x}) \equiv \psi_{d}\left(\frac{\left\|\mathbf{x}-\mathbf{c}_{i}\right\|}{\rho_{S}}\right)=\psi\left(\rho_{i}\right) .
$$

Here $\psi\left(\rho_{i}\right)$ is an RBF with support on the patch around the patch center $\mathbf{c}_{i}$ with a support radius $\rho_{S}$. The specific choice of $\psi_{d}{ }^{25}$ depends on the dimensions $d$ of the interpolant. For $d=2$ and $d=3 \psi_{d}$ is

$$
\psi=\left(4 \rho_{i}+1\right)\left(1-\rho_{i}\right)_{+}^{4},
$$

while for $d=4$ and $d=5$ it is

$$
\psi=\left(5 \rho_{i}+1\right)\left(1-\rho_{i}\right)_{+}^{5},
$$

(for more than 5 dimensions see $\left.{ }^{25}\right)$. The function $\psi_{2}\left(\rho_{i}\right)$ is shown in fig. 1. The specific choice of $\psi$ ensures derivatives of order two to be continuous at the patch boundaries where two or more patches overlap. The notation $(\cdot)_{+}$means that the function is non-zero only for a positive argument and thereby introduces the compact support. By choosing the support radius $\rho_{S}$ such that only a subset of the sample points is covered, the problem can

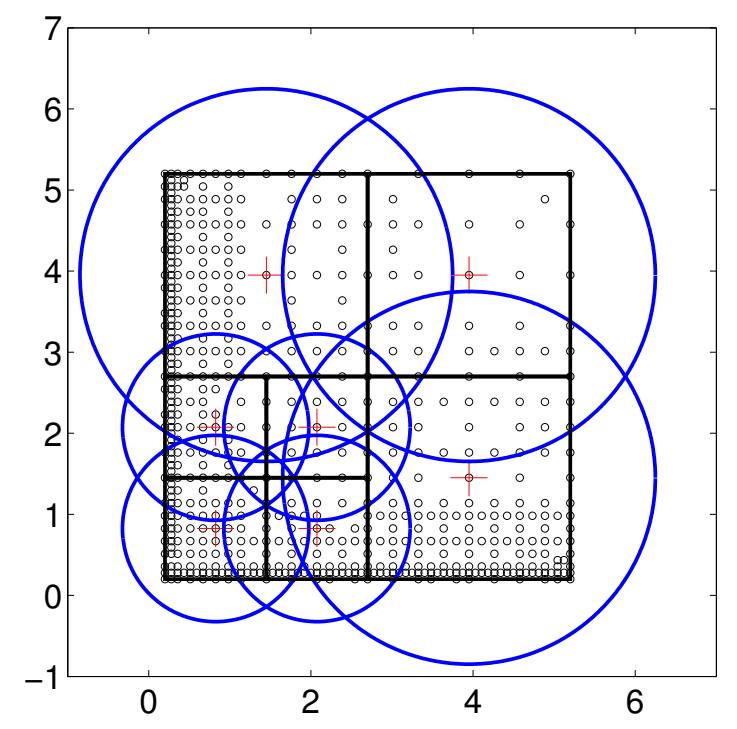

FIG. 2. Covering of a domain by circular patches ${ }^{26 \mid 27}$. The domain is first subdivided into boxes (black lines) such that the number of data points (black circles) in each box does not exceed a given limit. The patches are then formed as circles that that enclose each box (box and circle centers, red crosses). The circles (or hyperspheres in the general case) are enlarged by a fixed factor to ensure a sufficient overlap between the patches (blue circles).

be handled more efficiently for a large number of sample points. The size of the local (independent) PHS interpolation problems can now be chosen such that the linear systems of equations can be solved efficiently. A possible choice of patch covering is depicted in fig. 2. The specific choice of domain centers and patch sizes will be discussed in detail in sec. IIB3.

By using the combination of PHS and the PU method a hierarchical interpolation scheme is obtained which overcomes the scaling problems of a pure PHS interpolation 28. The splitting in localized patches also allows for an efficient parallel implementation and the extension to higher dimensions and larger number of sample points.

\section{B. Iterative refinement scheme}

The basic strategy to reduce the number of sample points needed, is to start out with a coarse grid and to 
refine locally on an as-needed-basis. The refinement procedure itself includes a scheme to generate new sample points in way that helps to reduce the amount of necessary function evaluations. We follow the ideas according to refs $26|29| 30$ describing a general scheme for the error scaling with step wise grid refinement. The error estimates determining the refinement are done by interpolating the error. This has the advantage that when testing for convergence, the function itself does not need to be evaluated.

\section{Refinement process}

In the following we describe the general procedure of the iterative refinement. The specific choice of sample points will be described in detail in the next section. Here the interpolation scheme from eq. (7) is used not only to approximate the function but also to approximate the error $e$ of the interpolant $s_{c}$ in the space between the sample points 30 . This has the advantage that the function itself needs not be evaluated to calculate the error in the new points which are considered for refinement.

For that purpose the error between the interpolant $s_{c}$ and the function $f$

$$
e^{k}(\mathbf{x})= \begin{cases}0 & \forall \mathbf{x} \in X_{c}^{k} \\ s_{c}^{k}(\mathbf{x})-f(\mathbf{x}) & \forall \mathbf{x} \in X_{e}^{k}\end{cases}
$$

is defined, where $k$ denotes the iteration index. Here the two different point sets $X_{c}^{k}$ and $X_{e}^{k}$ are used to define the error $e$. The sample points $X_{c}^{k}$ define $s_{c}$ and thus the error is zero in those points by definition. The error sample points $X_{e}^{k}$ are chosen from a subset of a finer grid than $X_{c}^{k}$ and are used to measure the error of $s_{c}^{k}$ with respect to $f$. An interpolant for the error $s_{e}^{k}$ can then be constructed from $e^{k}$ and the points $\left\{X_{e}^{k}, X_{c}^{k}\right\}$.

To refine further, a set of refinement points $X_{r}$ is chosen and the error in these points is estimated by $s_{e}^{k}$. To decide which function values are required to create an improved interpolant $s_{c}^{k+1}$, satisfying a given error bound $\epsilon$, the estimated error $\left|s_{e}\left(X_{r}\right)\right|$ and the calculated errors $\left|s_{c}\left(X_{e}\right)-f\left(X_{e}\right)\right|$ have to be checked against the error threshold value $\epsilon$. All points with an error exceeding $\epsilon$ are added to the set $X_{c}^{k+1}$ and eventually the next iteration can be performed. The procedure has converged if no further points have been added to $X_{c}$, or is terminated if a maximum number of iterations is reached.

The function $f$ itself is only evaluated at the points $X_{c}$ and $X_{e}$, but not at the refinement points $X_{r}$. Since the number of points grows polynomially during the refinement process the saving in terms of function evaluations of $f$ is substantial. The errors in the refinement points $X_{r}$ are estimated by interpolation and thus not necessary to evaluate directly. Hales et al. ${ }^{29}$ predicts linear convergence of the error with respect to the refinement level for a regular dense grid directly with PHSs. Even though this might not be the case for a non-regular grid as is produced by the algorithm presented here, the locally refined areas can be expected to show a similar convergence behavior.

\section{Generation of grid points}

In the following the specific choice of the sets of sample points $X_{c}, X_{e}$, and $X_{r}$ will be introduced. Possible schemes for choosing sample points are, e.g., low discrepancy points like Halton points ${ }^{31}$ or points that are suitable for polynomial approximations like Chebyshev points. Sparse grid methods ${ }^{32}$ combine results computed on a particular sequence of structured grids in order to get the final approximation. This type of technique has also been used together with RBFs 33 .

There are various types of point sets that have good properties for interpolation. However, here the choice of a simple regular grid greatly simplifies the creation and implementation of a systematic refinement scheme. The regular grid is expressed in terms of simple basis point sets which allow for a recursive refinement and a systematic splitting into local cells.

We use a dense grid to define the point sets, but use only those sample points that are necessary (as explained in the previous section). A simple choice is a so called product grid. Let $G^{(\ell)}(L)$ be a $d$-dimensional grid on the hyperrectangular domain $L$ defined by its side lengths $L_{i}$, $i=1,2, \ldots, d$, and let $\ell=\left(\ell_{1}, \ell_{2}, \ldots, \ell_{d}\right)$ be a multi-index describing the refinement. The step width between the grid points in a certain direction $i$ is then given by $L_{i} 2^{-\ell_{i}}$. The total number of grid points is given by $\left(2^{\ell}+1\right)^{d}$ if $\ell$ is equal for all $i$. In the local refinement scheme, a global point set of refinement level (depth) $\ell$ contains a subset of all points from the grid $G^{(\ell)}(L)$.

A hierarchical cell structure allows for organizing a local refinement, and can reduce the number of sample points, especially in higher dimensions. A cell $C$ is defined by its $2^{d}$ corner points, which in the following will be denoted by $V^{(0)}$, and forms the first basis point set for a cell (see fig. 3). To allow for local variations in the refinement we prefer to define the grid recursively by only using refinement levels $\ell=0$ and $\ell=1$ within a single cell. A fully refined cell, containing all the points of level $\ell=1$ can be split into $2^{d}$ new cells with refinement level $\ell=0$ forming the hierarchy of cells.

The local dense level 1 grid $V \equiv G^{(1)}(C)$ in a cell, as illustrated in fig. 3 is composed by $d$ different basis point sets, $V=\bigcup_{i=0}^{d} V^{(i)}$. The first point set $V^{(0)}$ represents corner points (as indicated above), $V^{(1)}$ represents edge centers, $V^{(2)}$ represents face centers, and so on. For convenience and to generalize the concept to an arbitrary number of dimensions we introduce a mathematical description for the basis points in a cell of unit side length. Let $r_{j}, j=1,2, \ldots, d$ be indices that can take the values 


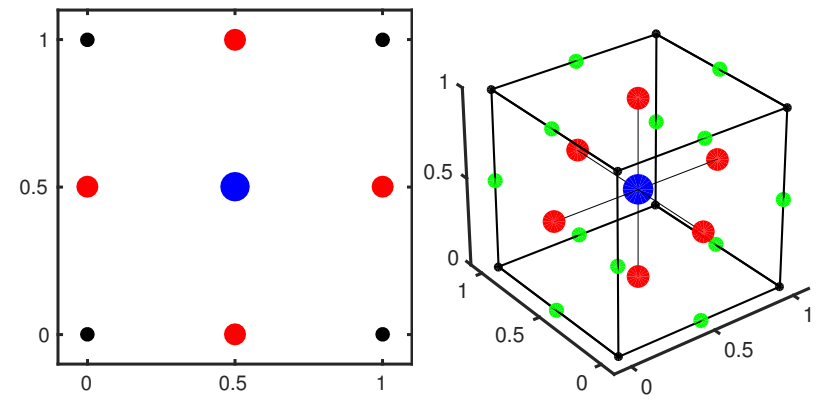

FIG. 3. Basis points for a product grid in a $2 \mathrm{D}$ (left) and a 3D cell (right). The functions are coded by color. 2D: black $V^{(0)}$, red $V^{(1)}$, blue $V^{(2)}$. 3D: black $V^{(0)}$, green $V^{(1)}$, red $V^{(2)}$, blue $V^{(3)}$. Note that both grids represent an $\ell=1$ refinement of a cell.

0,1 or 2 .

$$
b_{r}=\frac{1}{2}\left(r_{1}, \ldots, r_{d}\right) .
$$

In a grid consisting of several cells, we can also define the unique points within a cell as

$$
b_{r}^{\prime}=\frac{1}{2}\left(r_{1}, \ldots, r_{d}\right),
$$

where the indices $r_{j}$ can only take the values 0 or 1 .

A set of basis points $V^{(n)}$ is defined as all permutations of $b_{r}$ with $n$ odd indices $\left(n\right.$ indices $\left.r_{j}=1\right)$. This can be understood in terms of shifts. $V^{(0)}$ contains the unshifted corner points. One shift of half a cell length in any dimension gives a point in $V^{(1)}$, which is then an edge center. The single point in $V^{(d)}$ after $d$ shifts is always the cell center. The number of points in $V^{(n)}$ in a single cell is given by $\left(\begin{array}{l}d \\ n\end{array}\right) 2^{d-n}$. The basis point sets $V^{(n)}$ in two and three dimensions are illustrated in fig. 3 by differently colored dots.

To describe a locally refined grid a tree structure is used. The tree $\mathcal{T}^{(\ell)}$ of depth $\ell$ contains a subset of all points from the grid $G^{(\ell)}(L): \mathcal{T}^{\ell} \subseteq G^{(\ell)}(L)$. The tree itself is built from its nodes $\mathcal{T}_{i}^{j}, j=0,1, \ldots, \ell$, where $\mathcal{T}_{0}^{0}$ is the root node, and the range of $i$ depends on the number of cells at each level. Leaf nodes are denoted by $\mathcal{L}_{i}^{j}$. Each leaf node holds the information about its points. For an unrefined leaf cell, only the $V^{(0)}$ point set is present. Under refinement, further basis point sets are added until the correpsonding cell is fully refined, at which point the cell is split and another level of leaf nodes are created with the previous node as parent. The mapping between the nodes, leafs and the corresponding cells is sketched in fig. 4. A patch in the interpolation scheme covers a subtree with an appropriate number of associated points at its leaf nodes, see fig. 2.

For the refinement process the choice of sample and check points can now be derived from the point generation scheme described above. The set $X_{c}$ defining the interpolant is initially formed by the coarse grid $G^{\left(\ell_{0}\right)}(L)$.

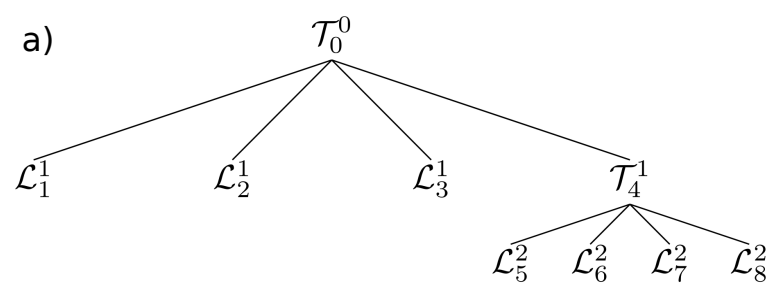

b)

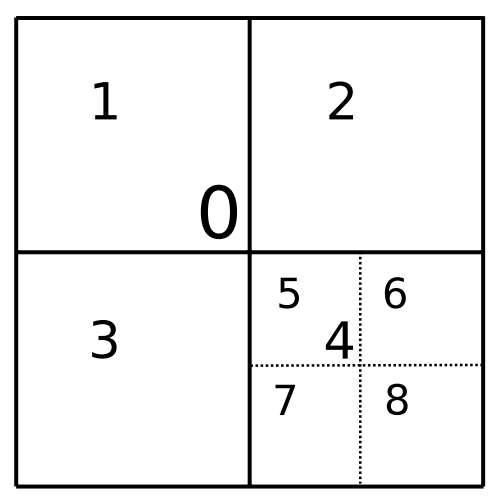

FIG. 4. Sketch of the cell structure and their subdivision. In (a) a tree with a local refinement depth of $\ell=2$ is shown. The corresponding grid is depicted in (b). The cells are linearly indexed: subcripts in (a) and numbers in (b).

The error check points $X_{e}$ are then all points in $V^{d}$ of all leafs nodes, namely the center points of all cells. The error estimate points $X_{r}$ are given by $V^{(d-1)}$. In case of $d>2$ the next step would be $X_{e} \in V^{(d-1)}$ and $X_{r} \in V^{(d-2)}$ and so on as long as $d-j>0$. A fully refined cell can then be split into $2^{d}$ new cells and the recursion depth increases. Note that the points $X_{c}$ used in the interpolant do not need to be all the points contained in $\mathcal{T}$, rather a subset creating a sufficient approximation $s_{c}$ of $f$.

\section{Algorithm}

We construct an algorithm which considers the special features of the PHS-PU scheme and incorporates the refinement process. We also address some practical issues in the algorithm. A formal outline of the algorithm is given in alg. 1. The main goal of the algorithm is to reduce the number of function evaluations of the function $f$ and at the same time provide a method which gives an reliable error estimate. In the final application $f$ will be a quantum chemistry program and $\mathbf{x}$ represents a molecular geometry in internal coordinates. Calculating $f(\mathbf{x})$ means to carry out a single point energy calculation, which is considered as the computationally expensive part. In essence the algorithm is designed to locally refine the grid as needed to represent the PES with a desired accuracy. Moreover, most applications only require 
a certain energy range of interest. This can be taken into account in the refinement scheme and provides a further improvement.

To start the refinement process a function $f(\mathbf{x})$ and initial set of points $X_{c}$ has to be chosen. It is assumed that a regular grid with a low refinement level $\ell_{0}$ is used. The level $\ell_{0}$ depends on the chosen order of the PHS and the size of the domain or the function $f$. The level $\ell_{0}$ should be chosen such that it has enough points for the linear system eq. (5) to be well posed and such that the qualitative features of $f$ are not undersampled. For most application $\ell_{0}=2$ (5 points in each directions) might be a good starting value.

The main refinement loop consist of two nested loops the outer loop refining $\ell$, while the inner loop refines the point set $V$ within the cells. In the inner loop the error function $e(\mathbf{x})$ from eq. $(12)$ is created from the sample points $X_{c}$ and the newly generated error check points $X_{e}$. For all points in $X_{e}$ the function $f$ has to be invoked in order to evaluate the interpolated error (eq. (12)). Error check points which are above the threshold join the set $X_{c}$ for the next iteration and contribute to a refined grid. Error checkpoints which are converged can remain in $X_{e}$, as this may improve the quality of the error estimator. The next step is to evaluate the approximate error $s_{e}(\mathbf{x})$ at the refinement points $X_{r}$ and add the points above threshold to $X_{e}$ for the next round. Note that to identify those points the function $f$ is not needed. This happens in the next step for the points in $X_{r}$ which are predicted to be not converged. After the inner loop is done nonconverged $X_{r}$ points are added to $X_{c}$. If there were no new $X_{c}$ points produced the refinement process can be considered as converged.

For example for a 3D system the initial choice of points in a single in the inner loop would go as follows. The point set $X_{c}$ consists of the all corner points of the cube $\left(V^{(0)}\right)$ and the error check points are the center points of the cube $\left(V^{(3)}\right) . V^{(d)}$ contains only a single point for each cell and has the largest possible distance from the corner points. The error estimate is then calculated for all points on the faces of the cube $\left(X_{r}=V^{(2)}\right)$. The error estimation $X_{e}$ is then improved with all non-converged points from $X_{r}$ In the next and last round the error is estimated for all points sitting on the edges on of the cube $\left(X_{r}=V^{(1)}\right)$.

After the iterations over the inner loop are finished, all active cells $\mathcal{L}$ which are subject to refinement are inspected. If a cell did not produce any new points it can be considered as locally converged and can be taken out of the refinement process. If all points of a cell are above a given energy threshold it can be also declared converged, since it is not in the region of interest. Also if all points in a cell produced invalid function values $f$ there is no need to refine them any further. This might however indicate severe problems of the electronic structure calculation in this region of space.

Now all the remaining leaf cells $\mathcal{L}_{r}$ are split up to produce a set of child cells which contain the newly generated

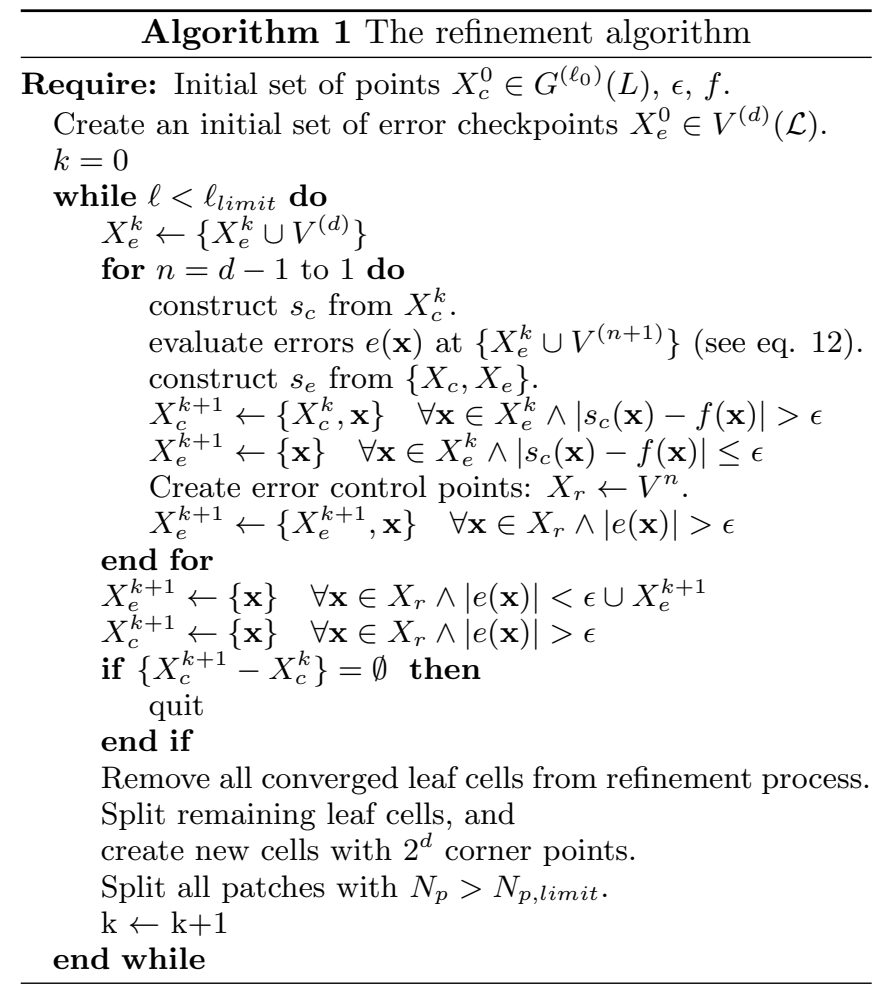

points of the last round. Since the number of points in $X_{c}$ used to build $s_{c}$ has increased it has to be checked that the number of points in the patches do not exceed a given limit. Otherwise the patches have to be subdivided accordingly. To keep the method simple a patch is built from a cell in the tree. Only the number of points in the cell (and its child cells) is counted to check the limits rather than the number of points in the final hypersphere enclosing the cell. The point limit $N_{p, l i m}$ has to be such that it contains enough points to ensure a well posed local PHS interpolant after splitting the patch.

After the procedure has reached convergence the result is a set of points $X_{c}$ which defines the interpolant $s_{c}$. There are still points in $X_{e}$ for which $f$ has been evaluated. These points can in principle be added to $X_{c}$ to improve the quality of $s_{c}$ at no further cost. We denote this merged point set by $X_{c}^{f}$. In the results we will also show a comparison of both variants.

\section{RESULTS}

To demonstrate the performance of the method a convergence study is conducted on a model function, which resembles a situation commonly found in molecules. Moreover an example for a PES produced by an electronic structure calculation will presented. 


\section{A. Model Potentials}

The workhorse for evaluating the method will be a Morse-potential:

$$
f(\mathbf{x})=\frac{1}{d} \sum_{i=1}^{d} \frac{g_{i}\left(x_{i}\right)}{g_{i}(0.2)}
$$

with

$$
g_{i}\left(x_{i}\right)=\left(1-\exp \left(-(1+0.1 i)\left(x_{i}-1\right)\right)\right)^{2}
$$

The function is chosen to be normalized between 0 and 1 in the investigated range $\left(x_{i} \in[0.2 ; 5]\right)$. Moreover the anharmonicities are chosen to be different for every dimension to break up symmetry. The Morse potential offers a simple but realistic scenario, to challenge the performance of the method. It provides high curvatures as well as flat areas which is useful to test the local refinement properties of the algorithm.

The interpolation errors are estimated by comparing at a set $X_{t}$ of 3000 random generated points versus the real function value. The figure of merit giving a measure for the reliability of the error estimates is the maximum error:

$$
\|E\|_{\infty}=\max _{\mathbf{x} \in X_{t}}\left|f(\mathbf{x})-s_{c}(\mathbf{x})\right| .
$$

A measure for the global quality is the average error:

$$
E_{a v g}=\frac{1}{N} \sum_{\mathbf{x} \in X_{t}}\left|f(\mathbf{x})-s_{c}(\mathbf{x})\right|
$$

\section{General convergence of the PHS-PU scheme}

In a first test, the convergence with respect to refinement is tested. No local refinement is used, but dense grids with different $\ell$ are evaluated. The errors here are not evaluated at random points but at the newly introduced points from the next refinement level $\ell+1$. In fig. 5 the result is shown for the 2D-Morse function from eq. (15). Here we also compare second (blue) and third order (green) PHS. In fig. 5(a) the maximum (solid lines) and mean errors (dashed lines) are shown. Comparing the maximum error and the average error shows that independent of the order, the rate of convergence for the average error is higher, and at $\ell=7$ the average error is about one order of magnitude better than the maximum error. It can be seen that the convergence rate for the third order PHS is significantly better than for $m=2$. The use of a $m=3$ PHS is thus preferred, since the computational cost is only slightly higher, but the result much better. At $\ell=7$ the maximum error is one order of magnitude better. In fig. 5 (b) the total number of grid points is plotted against the maximum error. This gives an impression of the increase of efficiency with increasing spline order. In principle even higher orders a)

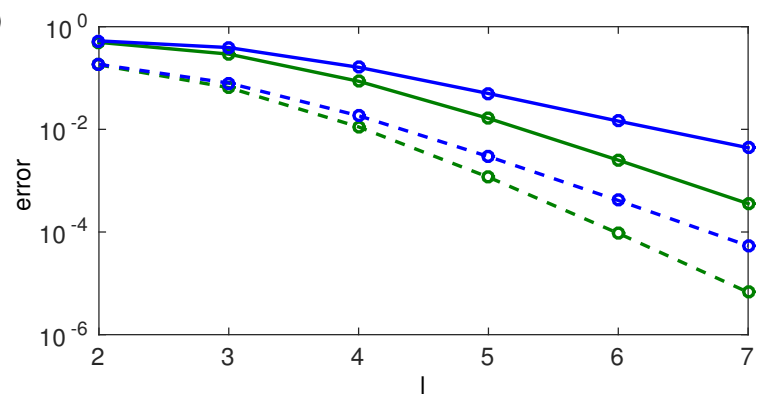

b)

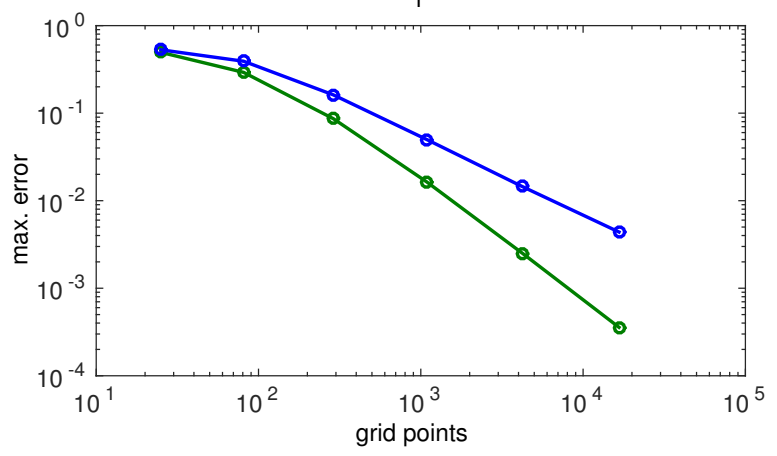

FIG. 5. Comparison of PHSs with order $m=2$ (blue) and $m=3$ (green) in two dimensions $(d=2)$ without adaptive refinement. In (a) the maximum error (solid line) and average error (dashed line) versus the grid refinement level $\ell$ is shown. In (b) the number of points vs. maximum error is shown.

might be used but with increasing length of the polynomial part $\mathcal{P}^{m}$ the minimum number of points needed to start the refinement algorithm is also increased. However, this becomes more challenging with an increasing number of dimensions.

\section{Demonstration of the local refinement process}

In fig. 6 the steps of the iterative refinement (alg. 1) are visualized for the Morse potential in two dimensions over all refinement steps until convergence is reached. We also make use of the feature to exclude regions of high energy on the PES to save function evaluations. The energy cutoff of $f_{\text {cut }}=0.33$ is indicated by the red line. The desired accuracy is set to $\epsilon=10^{-3}$ and the order of the PHS used is set $m=3$ from here on. Already in the $5 \times 5(\ell=2)$ grid of sample points, the basic qualitative features of the PES become visible. At $\ell \geq 3$ benefits of energy thresholds become visible. The flat areas in the lower right corner of the coordinate system are not refined any further. However they are described with sufficient accuracy and do not alter the qualitative shape of the PES. In the steps with $\ell>4$ the refinement is only needed at the outer regions close to the potential wall where the curvature of the function is high. It is noteworthy that the refinement points chosen here are mostly points on the edge of the cells and not their midpoints. This results in a local structure which is similar to a sparse grid 32 . 

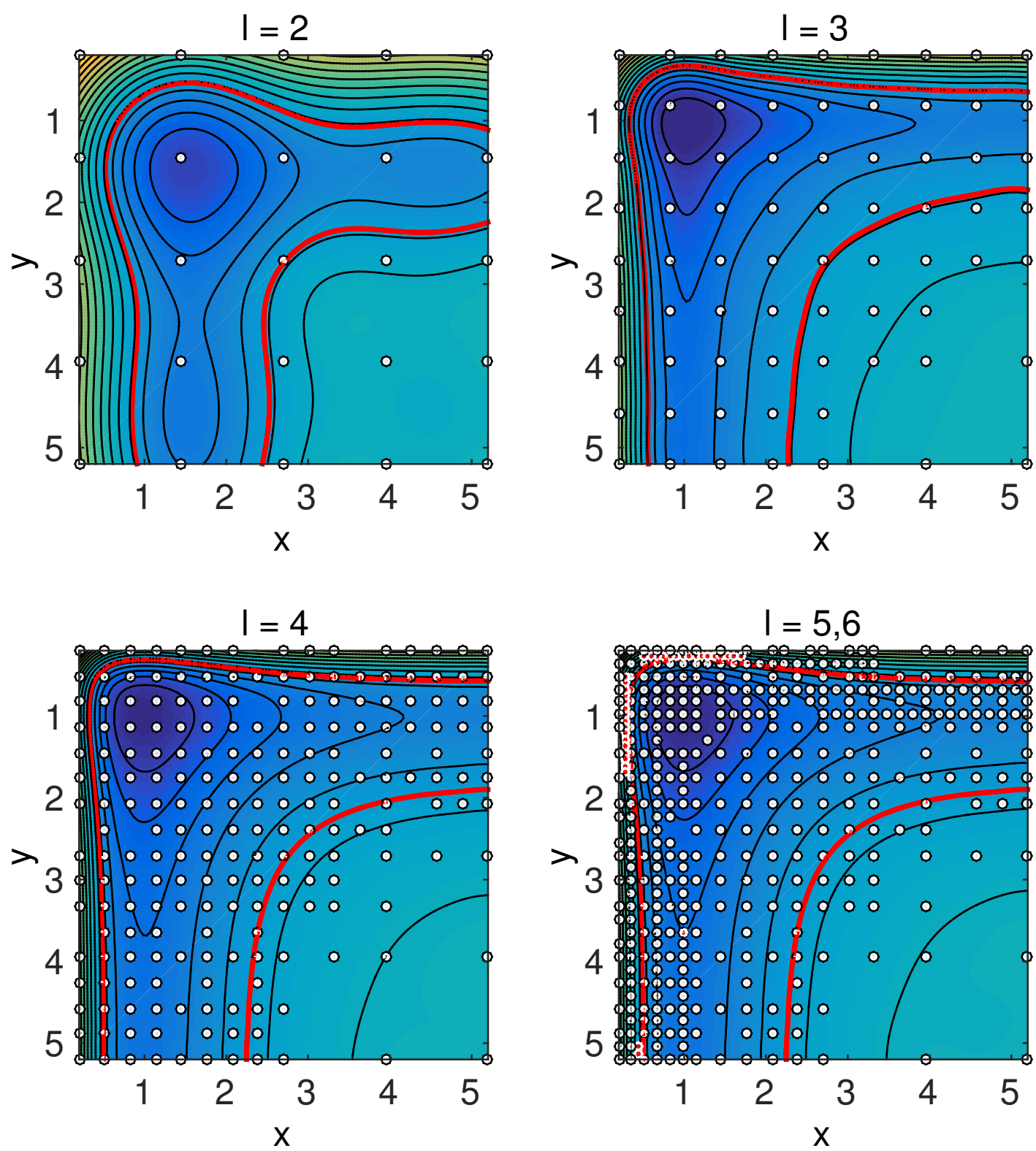

FIG. 6. Visualization of the iterations in the refinement process for different refinement levels $\ell$. The sample points of the interpolant are indicated by the white points. The red line indicates the contour of $f_{\text {cut }}=0.33$. The additional points introduced at the refinement level $\ell=6$ are indicated by the white, red filled points. The requested error is $\epsilon=10^{-3}$.

In the last step $\ell=6$ only a few new points are generated. They are located at the potential wall close to the minimum and support a steep region of high curvature and high curvature change rates. The result is a surface which is described by 428 sample points (the number of points in $X_{c}$ ). The total of amount function evaluations in this case was 799 (the number of points in $X_{c}^{f}$ ).

\section{Convergence with local refinement}

First we look at the reliability of the algorithm with respect to the requested error $\epsilon$ or the convergence criteria. The choice of grid points for error checking is an approximation which includes the assumption that the errors are largest in those points. For the following numerical test we make full use of the local refinement procedure and the PHS-PU scheme. The maximum number of points 


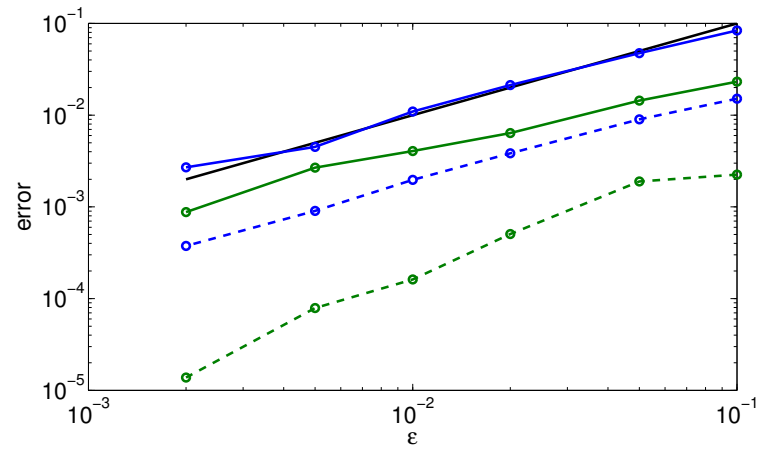

FIG. 7. Error measures vs. the requested error. Shown are the maximum errors (solid lines) and average errors (dashed lines) for a 3D Morse function. The two point set versions $X_{c}$ (blue) and $X_{c}^{f}$ (green) are shown. The black line indicates the requested limit $\epsilon$.

before a patch is split has been set to $N_{p, l i m}=300$.

In fig. 7 the maximum error $\|E\|_{\infty}$ (solid lines) and the mean error $E_{\text {avg }}$ (dashed lines) are plotted versus the requested error $\epsilon$ for the point sets $X_{c}$ and $X_{c}^{f}$. In the case of the maximum error it can be seen that the error can be larger than $\epsilon$, but is close to $\epsilon$ in the tested examples. However, $\|E\|_{\infty}$ scales accordingly with $\epsilon$ and gives a similar error. In contrast the average error is significantly better and well below the given error bound. The error study for a full grid refinement as shown in fig. 5 already indicates that due to the general convergence of PHSs the next refinement level yields a major improvement in the error. The dashed lines in fig. 7 represent the results when the calculated error control points are incorporated into the sample points $\left(X_{c}^{f}\right)$. In this example the maximum error is lowered by one order of magnitude compared with when using $X_{c}$. The average error is also improved significantly and is almost two orders of magnitude better than the requested error $\epsilon$. Its thus highly beneficial to use the full point set $X_{c}^{f}$. This means that the errors are significantly lower than $\epsilon$. In the following the $X_{c}^{f}$ point sets are used in the results if not otherwise stated.

Another interesting question is the scaling behavior with respect to the number of dimensions. The underlying interpolation method and the refinement process are designed to work for any number of dimensions $(d \geq 2)$. In the following we interpolate on a smaller domain such that $x_{i} \in[0.3 ; 3.3]$ and compare the scaling for $d=2,3,4$. In fig. 8 the maximum error is plotted against the average number of points per dimension $N^{1 / d}$ which is proportional to the average fill distance of points 15 . The dashed lines in fig. 8 indicate the expected third order convergence $e^{15}$ (p. 129). The improvement of the maximum error is defined by the following equation:

$$
\lim _{n \rightarrow \infty}\left\|E_{n+1}\right\|_{\infty}=C \frac{\left\|E_{n}\right\|_{\infty}}{N_{n}^{r / d}},
$$

where $r$ is the rate of convergence. It can be seen that

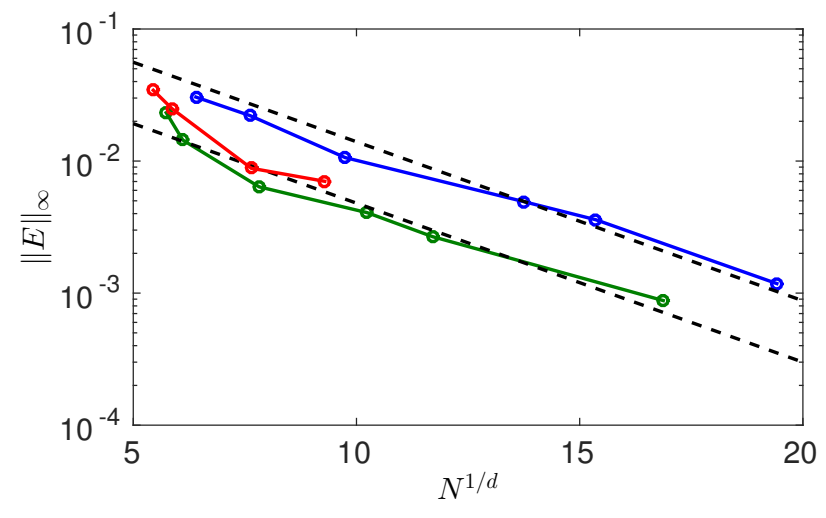

FIG. 8. Maxmimum error vs. the number of total points $\left(X_{c}^{f}\right)$ per direction. Comparison of 2D (blue), 3D (green), and 4D (red). The dashed line indicates third order convergence.

for all dimensions shown third order convergence can be achieved. For 3 and 4 dimensions the necessary number of points per direction is even decreased. This result provides valuable information and can be used to extrapolate to the necessary number of points needed for a certain accuracy by using a coarse interpolant.

\section{Efficiency of the local refinement}

The iterative refinement process is designed to use localized error estimates to decide where refinement of the sampling grid is needed. By only refining on an asneeded-basis the method is expected to be more efficient than an overall refinement on a dense grid. The efficiency of the local refinement is evaluated by the maximum refined level $\ell_{\max }$ a calculation has reached. We then define the efficiency as the ratio of the actual number of sample points needed per dimension $N^{1 / d}$ and the number of points per dimension generated in a full dense grid of level $\ell_{\max }$ :

$$
R=\frac{N^{1 / d}}{\left(2^{\ell_{\max }}+1\right)}
$$

Figure 9 shows the efficiency as a function of the maximum error for $d=2,3,4$. With an increasing number of dimensions the saving in terms of sample points to represent the PES increases.

\section{B. Molecular PES from ab intio data}

To demonstrate the interpolation method on a more realistic 3-dimensional example, we test it against an example as it might used in a reactive scattering calculation 17 . Here the PES for the backside attack of the nucleophilic substitution reaction $\mathrm{Cl}^{-}+\mathrm{CH}_{3} \mathrm{~F} \rightarrow \mathrm{ClCH}_{3}+\mathrm{F}^{-}$is calculated. The single point energies are calculated at the 


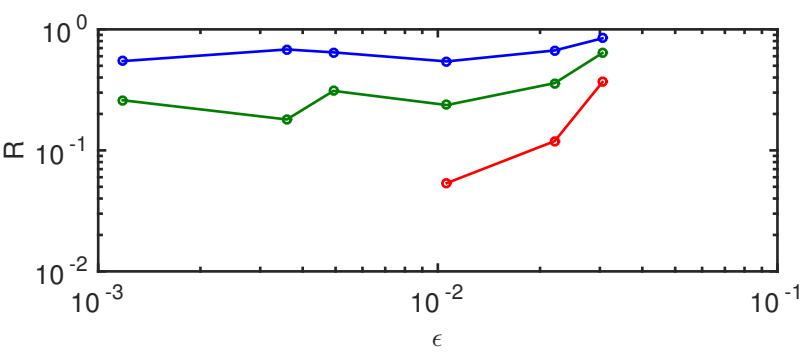

FIG. 9. Efficiency of the refinement method vs. the maximum error. Comparison of $d=2$ (blue), $d=3$ (green), and $d=4$ (red). Smaller numbers are better.

TABLE I. Ranges for the calculation of the PES

\begin{tabular}{lrr}
\hline \hline variable & $\min$. & $\max$. \\
\hline$r_{C-C l}$ & $1.1 \AA$ & $5.1 \AA$ \\
$r_{C-F}$ & $0.8 \AA$ & $4.8 \AA$ \\
$\theta$ & $40^{\circ}$ & $100^{\circ}$ \\
\hline \hline
\end{tabular}

$\mathrm{MP} 2 / 6-311+\mathrm{G}^{*}$ level of theory with the ab initio program package Gaussian09 ${ }^{34}$. The active coordinates are the carbon-chlorine distance $r_{C-C l}$, the carbon-fluorine distance $r_{C-F}$, and the umbrella angle $\theta$ of the $\mathrm{CH}_{3}$ group, where $\theta=90^{\circ}$ corresponds to a planar $\mathrm{CH}_{3}$ group. The system is assumed to preserve $\mathrm{C}_{2 \mathrm{v}}$ symmetry during the course of the reaction and thus $\mathrm{Cl}, \mathrm{C}$, and $\mathrm{F}$ are in a linear configuration. The range over which the PES is calculated is given in tab. I]

The iterative refinement process according to alg. 1 for the PES is performed to obtain a set of sample points and their corresponding values defining the 3D-PES interpolant. Each function evaluation $f\left(r_{C-C l}, r_{C-F}, \theta\right)$ calls the ab initio program with the corresponding molecular geometry and returns the corresponding energy value. The only tunable input parameters used here are the relative error tolerance $(\epsilon=0.01$ Hartree $)$, and the cutoff value (0.2 Hartree, with respect to minimum), which are chosen to obtain a fast convergence of the procedure and only focus on the relevant energy range. Running the automated interpolation procedure results in a set of 5008 points (the $X_{c}^{f}$ point set). The interpolated PES is shown in Fig. 10 in form of 2D slices for umbrella angles corresponding to the reactants, the transition state and the products.

To evaluate the errors the interpolant is tested against a set of 4159 randomly generated ab initio points, which are below cutoff in the relative energy region. The maximum error found is $\|E\|_{\infty}=1.75 \cdot 10^{-3}$ Hartree which is on the order of magnitude below the requested error bound $\epsilon$, which confirms the trend found in fig. 77. The average error found here is $E_{\text {avg }}=7.51 \cdot 10^{-5}$ Hartree, meaning that the average discrepancy between the interpolant and the ab initio calculation in this example is only $2.04 \mathrm{meV}$. The maximum refinement depth that has been reached for some cells during the refinement process was $\ell=6$. A full, dense grid with $\ell=6$ requires 65 sample points for each dimension resulting in a total of 274625 sample points. The local refinement procedure thus provides a interpolant with only $1.8 \%$ of the sample points when compared to a dense grid.

\section{CONCLUSION}

In this paper we have presented an adaptive interpolation algorithm for the efficient creation of approximate global PESs. The combination of a PHS scheme with a PU scheme allows to efficiently handle a larger number of sample points. Ill-conditioning of the interpolation matrix is avoided. We have demonstrated the error scaling for a the adaptive, local refinement algorithm. The error estimation and local refinement method presented within this approach have been tested with data sets of 2-4 dimensions. The empirical study of the errors confirms that the error bounds are reliable. It has been found that the average observed errors are lower by approximately one order of magnitude than the requested error. The local refinement makes the scheme highly efficient and places sample points on an as needed basis. The additional energy cutoff criteria makes the algorithm even more efficient. The number of required sample points which needed to be evaluated in the chosen examples are between 1-10\% compared to a grid of sampling points of predefined density. Using this method in combination with state of the art ab intio methods allows for an efficient calculation of high quality global PESs.

Even though a direct calculation of higher dimensional ( $>6$ dimension) seems not be feasible with the present method, the PES for higher dimensional problems can be approximated by a many-body expansion $\frac{135+37}{13}$ as it is commonly used with e.g., vibrational configuration interaction methods 38

$$
\begin{aligned}
V\left(q_{1}, \ldots, q_{N}\right) & =\sum_{i} V_{i}\left(q_{i}\right)+\sum_{i<j} V_{i j}\left(q_{i}, q_{j}\right) \\
& +\sum_{i<j<k} V_{i j k}\left(q_{i}, q_{j}, q_{k}\right)+\ldots
\end{aligned}
$$

where $V_{i}, V_{i j}, V_{i j k}$, etc. are the 1-mode, 2-mode, 3-mode contributions respectively. These individual components are in itself $1,2,3, \ldots$ dimensional potential energy surfaces, which can be generated in a efficient way with the presented method (Eqs. 1, 7, and Alg. 1).

\section{ACKNOWLEDGMENTS}

M.K. acknowledges support through the Centre of Interdisciplinary Mathematics (CIM), Uppsala University. The computations were performed on resources provided by SNIC through Uppsala Multidiciplinary center 
a)

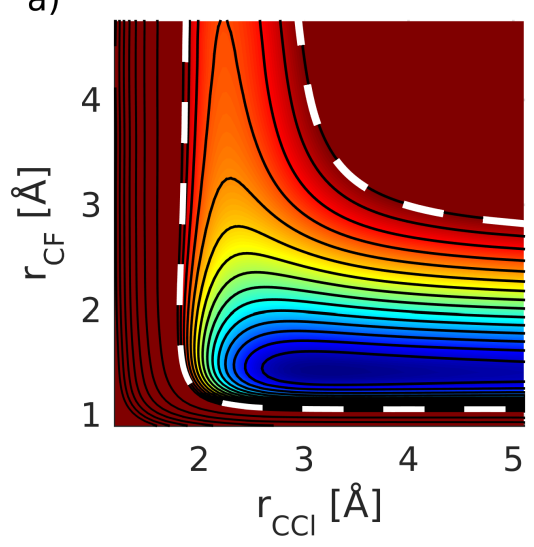

b)

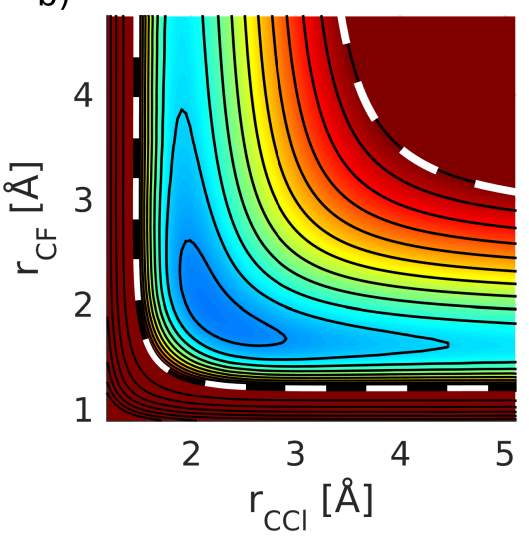

c)

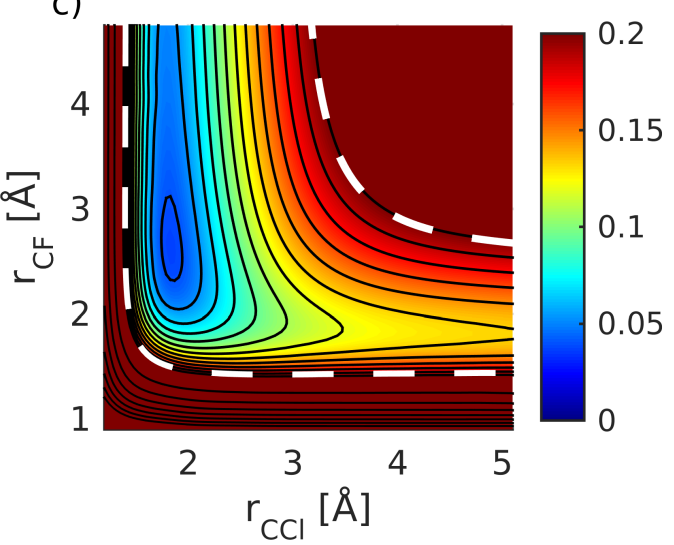

FIG. 10. 2D slices through the interpolated PES of the nucleophilic substitution reaction $\mathrm{Cl}^{-}+\mathrm{CH}_{3} \mathrm{~F} \rightarrow \mathrm{ClCH}_{3}+\mathrm{F}^{-}$for different values of $\theta$ : (a) $71.5^{\circ}$, (b) $95^{\circ}$, and (c) $108^{\circ}$. The color scale is truncated above 0.2 Hartree (5.44 eV) (indicated by the white dashed line). The spacing between the black, solid contour lines is 0.11 Hartree for energie values $>0.2$ and 0.013 Hartree for energie values $<0.2$ Hartree.

for Advanced Computational Science (UPPMAX) under Project snic2013/1-267. The research of A.H. was partially supported by the National Science Foundation (grant DMS-1318427).

${ }^{1}$ R. Marquardt and M. Quack, Global Analytical Potential Energy Surfaces for High-resolution Molecular Spectroscopy and Reaction Dynamics (Wiley, 2011), pp. 511+, ISBN 9780470749593.

${ }^{2}$ D. J. Tannor, Introduction to Quantum Mechanics: A TimeDependent Perspective (University Science Books, 2006), ISBN 1891389238.

${ }^{3}$ A. J. C. Varandas, Adv. Chem. Phys. pp. 255-338 (2007).

${ }^{4}$ S. Manzhos, R. Dawes, and T. Carrington, Int. J. Quantum Chem. 115, 1012 (2015).

${ }^{5}$ J. Behler, Int. J. Quantum Chem. 115, 1032 (2015).

${ }^{6}$ D. Shepard, in Proceedings of the 1968 23rd ACM National Conference (ACM, New York, NY, USA, 1968), ACM '68, pp. 517524.

${ }^{7}$ O. Tishchenko and D. G. Truhlar, J. Chem. Phys. 132, 084109+ (2010).

${ }^{8}$ G. E. Moyano and M. A. Collins, J. Chem. Phys. 121, 9769 (2004).

${ }^{9}$ M. Majumder, S. A. Ndengue, and R. Dawes, Mol. Phys. 114, 1 (2016).

${ }^{10}$ T. Ishida and G. C. Schatz, Chem. Phys. Lett. 314, 369 (1999).

${ }^{11}$ D. R. McLaughlin and D. L. Thompson, J. Chem. Phys. 59, 4393 (1973).

${ }^{12}$ C. Xu, D. Xie, D. H. Zhang, S. Y. Lin, and H. Guo, J. Chem. Phys. 122, 244305+ (2005).

${ }^{13}$ T. Hollebeek, T.-S. Ho, H. Rabitz, and L. B. Harding, J. Chem. Phys. 114, 3945 (2001).

${ }^{14}$ D. Opalka and W. Domcke, J. Chem. Phys. 138, 224103+ (2013).

${ }^{15}$ G. E. Fasshauer, Meshfree Approximation Methods with Matlab (World Scientific, 2007), ISBN 978-981-270-633-1.

${ }^{16}$ D. Geppert, A. Hofmann, and R. de Vivie-Riedle, J. Chem. Phys. 119, 5901 (2003).

${ }^{17}$ M. Kowalewski, J. Mikosch, R. Wester, and R. de Vivie-Riedle, J. Phys. Chem. A 118, 4661 (2014).

${ }^{18}$ A. Szabo and N. S. Ostlund, Modern Quantum Chemistry (Dover Publications, New York, 1996).
${ }^{19} \mathrm{H}$. Wendland, in Approximation Theory X: Wavelets, Splines, and Applications (2002), vol. 473, pp. 473-483.

${ }^{20}$ J. Duchon, RAIRO Analyse Numérique 10, 5 (1976).

${ }^{21} \mathrm{~J}$. Duchon, in Constructive Theory of Functions of Several Variables, edited by W. Schempp and K. Zeller (Springer Berlin Heidelberg, 1977), vol. 571 of Lecture Notes in Mathematics, pp. 85-100, ISBN 978-3-540-08069-5.

${ }^{22}$ R. K. Beatson and W. A. Light, IMA J. Numer. Anal. 17, 343 (1997).

${ }^{23}$ R. K. Beatson, M. J. D. Powell, and A. M. Tan, IMA J. Numer. Anal. 27, 427 (2007).

${ }^{24}$ R. K. Beatson, J. B. Cherrie, and D. L. Ragozin, SIAM J. Math. Anal. 32, 1272 (2001).

${ }^{25}$ H. Wendland, Adv. Comput. Math. 4, 389 (1995).

${ }^{26}$ T. A. Driscoll and A. R. H. Heryudono, Comput. Math. Appl. 53, 927 (2007).

${ }^{27}$ A. Safdari-Vaighani, A. Heryudono, and E. Larsson, J. Sci. Comput. 64, 341 (2015).

${ }^{28}$ R. Cavoretto and A. De Rossi, Comp. Math. Appl. 67, 1024 (2014).

${ }^{29}$ S. J. Hales and J. Levesley, Numer. Algorithms 30, 1 (2002).

${ }^{30} \mathrm{~A}$. Iske and J. Levesley, 39, 187 (2005).

${ }^{31}$ J. H. Halton, Numer. Math. 2, 84 (1960).

${ }^{32}$ T. Gerstner and M. Griebel, in Encyclopedia of Quantitative Finance, edited by R. Cont (John Wiley and Sons, 2010).

${ }^{33}$ E. H. Georgoulis, J. Levesley, and F. Subhan, SIAM J. Sci. Comput. 35, A815 (2013).

${ }^{34}$ M. J. Frisch, G. W. Trucks, H. B. Schlegel, G. E. Scuseria, M. A. Robb, J. R. Cheeseman, G. Scalmani, V. Barone, B. Mennucci, G. A. Petersson, et al., Gaussian09 Revision D.01, gaussian Inc. Wallingford CT 2009.

${ }^{35}$ H.-J. Werner, P. J. Knowles, G. Knizia, F. R. Manby, M. Schütz, et al., Molpro, version 2012.1, a package of ab initio programs (2012), see http://www.molpro.net.

${ }^{36}$ G. Rauhut, J. Chem. Phys. 121, 9313 (2004).

${ }^{37}$ T. Hrenar, H.-J. Werner, and G. Rauhut, J. Chem. Phys. 126, $134108+(2007)$.

${ }^{38}$ M. Neff and G. Rauhut, J. Chem. Phys. 131, 124129+ (2009). 